\title{
The use of animals in research
}

\author{
Medical progress depends on it
}

While the whole population (of animals and humans) benefits from biomedical research, doctors are - in the language of the new NHS - the purchasers of this activity and have a particular and powerful interest in seeing that it is well done. When, therefore, the research enterprise is under threat-as it is from the animal lobby's concerted campaign - it must be right for the profession to make its views and its legitimate concerns publicly known, and the BMA's decision to debate the subject at this year's annual representative meeting is very welcome.

Advances in the biomedical sciences generally come from combining results of experimentation at all levels from the molecular to the clinical. Advances in molecular biology have revolutionised the experiments that can be done in subcellular systems and those using bacteria or yeasts as test tubes. The growth of modern cell biology and of tissue culture techniques has similarly transformed what can be learnt from living cells in vitro. Although these advances have changed the questions that need answering in the intact organism, be it animal or human, they have not totally replaced (and do not look likely to replace) in vivo experimentation. Much that goes on in vivo depends on "geographical" organisation - be it in the nervous system, the immune system, or the cardiovascular systemand this cannot be studied adequately ex vivo. The techniques of in vivo genetic manipulation are enormously powerful and will allow research workers to study directly the functions of genes in vivo, which has previously been impossible or dependent on rare spontaneous mutants. Such experiments are already showing the greatest potential for understanding of genetic diseases and for innovative treatments such as gene therapy. ${ }^{1}$ This type of experimentation cannot be done exclusively in humans.

A backward look at the striking advances that have come from medical science shows that virtually all are based on the full range of available experimentation and could not have happened without the use of experiments in animals and humans. The development of vaccines; the discovery of antibiotics, hormones, and vitamins; and the introduction of organ transplantation are just a few examples. Pasteur's introduction of rabies vaccination after exposure was one of the earliest triumphs of research on vaccines. Interestingly, one of the most recent successes of research on vaccines again concerns rabies - this time it is the vaccination of animals in the wild by dropping meat treated with recombinant vaccinia virus containing rabies antigens. ${ }^{2}$ This promises to eradicate rabies in the wild and to eliminate the threat that the disease poses to humans: a fine example of research benefiting all animals, not just those exploited by humans.

The ethics of research in the intact organism have always been kept under careful scrutiny in Britain, and this scrutiny has recently become more rigorous. Curiously, experimentation on laboratory animals has generated so much more controversy than clinical research on human beings. For clinical experimentation the ethics are in general well understood, both by the experimenter and by the subject. The consent of the subject is required, and ethics committees specially set up for the purpose have to approve the experimental protocols. The use of animals in research is different in so far as the animal cannot give consent and animal experimentation has long been controlled by legislation and subject to an inspectorate provided by the Home Office. None the less, there is vocal opposition to the use of animals, even in the most exemplary medical research, on the grounds that it infringes the rights of animals.

The proposition that rights (and their inescapable counterpart, duties) - both of which are essentially human concepts - can be applied to other than human beings is problematical. Does a tree have a right not to be cut down? Does the right of a lion to eat a zebra take precedence over the right of the zebra not to be eaten? Do trees or lions or zebras have any duties at all? What is neither problematical nor even contentious is that humans certainly have duties towards animals, which include respect for animal life and avoiding cruelty and the infliction of suffering. These duties are not restricted to the animals used in medical research but extend equally to animals that are farmed for food or other products, that are kept for their labour or as companion animals, and even that are exterminated as vermin. It is worth pointing out that more than $80 \%$ of animals used for medical research are short lived rodents (mice and rats), which fall into this last category. If one believed in the Pythagorean scheme of reincarnation into other forms of animal life and it was one's fate to be reincarnated as a rodent it would be wise to choose to be reincarnated as a laboratory rodent rather than one living in the wild. Life would on average be longer and more likely to be free of pain and hunger.

\section{President,}

Royal College of Pathologists,

London SWIY 5AF

1 Miller AD. Human gene therapy comes of age. Nature 1992;357:455-60.
2 Wandeler AI. Control of wildlife rabies: Europe. In: Campbell JB, Cha

2 Wandeler AI. Control of wildlife rabies: Europe. In: Campbell JB, Charlton KM, eds. Rabies. Boston: Kluwer Academic, 1988:365-80. 